\title{
Online diagnostics for laser cleaning of artworks
}

\author{
K. Dickmann, M. Lentjes \\ Laser Centre FH Münster (LFM), University of Applied Sciences, Münster/Germany, laserlab@fh-muenster.de
}

J. Meijer

Faculty of Engineering Technology, University of Twente, Enschede/The Netherlands, j.meijer@wb.utwente.nl

In order to avoid over-cleaning during laser assisted restoration of artworks, several techniques have been developed. As a powerful method Laser Induced Breakdown Spectroscopy (LIBS) has turned out being a promising candidate (e.g. Zafiropulos et.al.). Monitoring during laser cleaning using high resolutions LIBS is characterised by the comparison of selected single elemental peaks. However, the acceptance in practise of restoration is weak due to the complex spectroscopy technique (a), lacking spectroscopy experience of the operating restorer (b) and high investment cost related to the cleaning laser system (c).

To overcome the listed drawbacks we have used novel miniature fibre optic spectrometer technology with CCDdetector ("Ocean Optics HR2000"). Our system offered a wide spectral range $200-1100$ nm at low $\lambda$-resolution $(2 \mathrm{~nm})$. In contrast to approved LIBSpectroscopy using ICCD detectors, sensitivity and $\lambda$-resolution is much less. However, it was found out that for monitoring during laser cleaning the complete (low resolution) spectrum may be used as an individual "fingerprint" of the layer just being laser irradiated.

In practise of laser cleaning a permanent comparison of the spectrum from the ablated layer with a reference spectrum of the layer to be preserved is necessary. In order to simplify this procedure for application in practise we used "Pearsons Linear Correlation Method". The coefficient $r$ quantifies the correlation between the spectra. A value of $r=1$ represents a correlation of $100 \%$. The $\mathrm{x}$ data set is given by the spectrum of the irradiated layer while $\mathrm{y}$ is the data set of the reference spectrum. Index "i" represents the pixel number of the CCD-array

$$
r=\frac{\sum_{i=1}^{n}\left(x_{i}-\bar{x}\right)\left(y_{i}-\bar{y}\right)}{\sqrt{\sum_{i=1}^{n}\left(x_{i}-\bar{x}\right)^{2}} \sqrt{\sum_{i=1}^{n}\left(y_{i}-\bar{y}\right)^{2}}}
$$
( $\mathrm{i}=1 \ldots 2048$ ). Using this correlation method, intensity fluctuations of the spectra (e.g. caused by pulse to pulse instabilities of the laser) do not affect the coefficient $r$. The period for calculation of the correlation coefficient $r$ is only $<15 \mathrm{~ms}$ (including data transfer from CCD-pixels). Thus, related to repetition rates of laser pulses $<10 \mathrm{~Hz}$ (commonly used for laser cleaning) quasi online monitoring is possible.

For testing our miniature fibre optic setup we used $\mathrm{KrF}$-excimer laser radiation $(248 \mathrm{~nm}, 35 \mathrm{~ns})$ and an artificial complex multi layer sample (silver acrylate, black acrylate, adhesive, paper). In this specific case of demonstration we used 3 different reference spectra: "black", "adhesive", "paper". The corresponding correlation coefficients $r$ calculated during the ablation versus ablation pulse number are shown in fig. 1 . It can be seen that $r$ reaches maximum $(\approx 1)$ at the transitions silver/black, black/adhesive and adhesive/paper (indicated by the arrows). In first attempts successfully we used our rugged and compact system for online monitoring during laser cleaning of glass covered with strong encrustation. Monitoring was carried out by permanent estimation of $r$. Closedloop laser cleaning is in preparation.

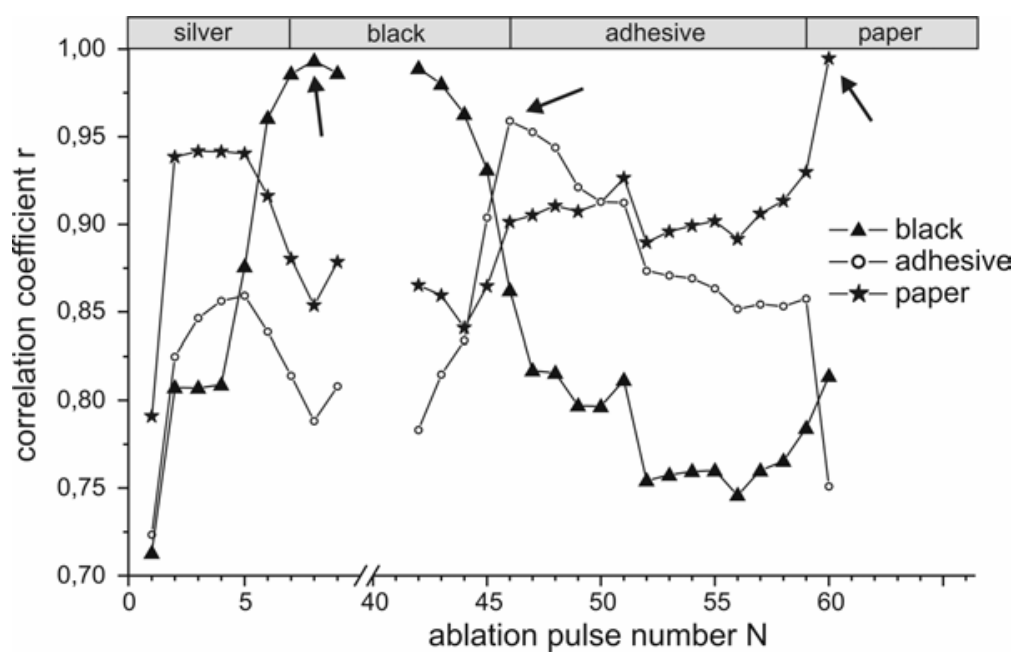

Fig. 1.: Capability of the miniature LIBS system for the detection of individual layers during laser ablation/cleaning. Correlation coefficients $r$ related to the reference spectra "black", "adhesive" and "paper" with increasing penetration into the multi layer test sample. The arrows indicate the maximum of $r$, always appearing at the transitions. 